\title{
Discrete manufacturing systems PLM control platform and its operation mode in the Internet of Things

\author{
WANG Zhiguo ${ }^{1,2}$, WANG Fei ${ }^{1}$, YANG Shanlin ${ }^{2}$
}

\author{
${ }^{1}$ School of management engineering, Anhui Polytechnic University, 241000, Wuhu , Anhui, \\ China \\ ${ }^{2}$ School of Management, Hefei University of Technology, 230009, Hefei, Anhui, China.
}

\author{
Keywords: Discrete manufacturing systems; PLM control platform; manipulated integration \\ platform; Integrated of Things
}

Abstract. For adapting the global Internet of Things, one of important things is building product lifecycle management (PLM) visualization control platform. The goal of visualization of PLM is to build a information integration platform. The information platform includes all kinds of demand information for all kinds of faculties from the managers to operators. The information is pushed to demanders freely and initiatively including control suggestion information, effectiveness prediction information and simulation information, etc al.. The information platform could provide bi-direction communication between manufacturer and manager. In this paper discrete manufacturing system is taken as studying object. A set of technology system of visualization manipulation of PLM is established by the techniques and methods of the Internet of Things and visualization management. The technology system includes the manipulation integration model, controlling techniques, dashboard architecture, prediction and simulation of decision effectiveness, rapid construction and reconfiguration based on soft component model-driven. The system adapts the changes of internal structure and external environment of PLM.

\section{Introduction}

Nowadays manufacturing enterprises are facing with increasing competitive pressures, product delivery cycles are becoming shorter and shorter, more and more high quality,lower and lower prices. Under these challenges the companies have taken a variety of means and measures to improve and enhance their own competitiveness constantly. Various means, measures and tools are use to improve the enterprise's rapid response capabilities. All of these are inseparable from monitoring, diagnosing, optimizing and controlling the production process and operational status in real-time. In order to achieve the goal, it is necessary to describe the modeling for the state of system and process, determine parameters of the state and the real-time acquisition, analysis methods and means of its values. Because of the limitations of real-time data acquisition, the analysis and diagnosis methods, the model simulation and optimization are studied on discrete manufacturing systems PLM control platform are progressing slowly, the emergence of Internet of Things is to provide new ideas and approaches to the research of this topic.

Concerned the management of Internet of Things, the visualization of product lifecycle management, controlling modeling, and controlling simulation, are having been attracted a lot of researchers. For example, Professor Qi Guoning of Zhejiang university studied the project management of product life cycle management ${ }^{[1]}$. Wang Chengen of Northeastern university studied the modeling and management of product life cycle ${ }^{[2]}$. Josef Scho ttner of Munich industrial university studied manufacturing enterprise's product data management principles, concepts, strategies ${ }^{[3]}$, and so on. In the present research, the research object based on the mathematical model control method and additional assumptions are often far away from the actual situation and it is difficult to be used in complex PLM effectively. For the limitations of conventional intelligent method and simulation technology, some technology companies have invested considerable human and financial resources, combined with the particular goals in the PLM, promote research and development of automation technology, have achieved some practical results, but these studies are lack of universality ${ }^{[4-6]}$. Particularly it is hard for production conditions changed frequently in 
manufacturing enterprises. So it is urgent that actual demand of complex PLM control and optimization is studied combined with the intelligent method, optimization theory. It is needed to study optimization arithmetic of high performance, confirm and apply it in the typical complex PLM, lay a solid foundation for the development of PLM modeling and optimization software which has our country's independent property rights in the networking environment, in order to improve the overall level of the automation of manufacturing industry in China.

\section{Discrete manufacturing PLM platform control system and control integration model}

The development and operator of discrete manufacturing system of PLM control platform ,can be combined with the specific types and characteristics of manufacturing services, after determining the service areas, using the key technologies such as core theory, standards, system framework, the common engine, common management tools, services, and operation mode, develop and implement discrete manufacturing system PLM control platform of the specific industry and the distinctive region. The function of discrete manufacturing system PLM control platform includes the management of unified user,registration and publish of manufacturing resources,publish of manufacturing requirements, and registration center,transaction system,transaction management,and business management of manufacturing services,credit assessment and analysis of business, industry knowledge accumulation and service network community, system management of control platform , and so on. Under the support of the platform, manufacturing resources can be realized sharing and integration. For example, the surplus production capacity of the numerical control machine tools, test equipment, and analysis software in certain company, these resources could be virtualized clouds access through the platform based on the network to provide manufacturing services. At the same time, the discrete manufacturing system with service needs, through the platform can be widely looking for manufacturing resources required, and customize the manufacturing services according to the need with low cost .

Discrete manufacturing system PLM control platform system structure is from the angle of the service achieving,settle and plan the level of the platform structure and logic relationship, to construct the architecture blueprint of discrete manufacturing of PLM control platform system ${ }^{[7]}$. Combine existing research results of networked manufacturing control platform with the connotation of discrete manufacturing system PLM control platform, set up a kind of system architecture, which is in the discrete manufacturing system PLM control platform under the environment of Internet of Things.

The interpretation of modules shows: (1) basic support layer is mainly to provide cloud control platform database, network which are basic support environment(2)platform integrated operation environment is mainly provide a platform operation security, monitoring and management of the basic environment. (3) the platform layer provides a series of tools, support the manufacturing resources and the demand of convenient registration, issued, search matching, trade, and business management, evaluate both sides credit and create knowledge community, etc. Layer (4) manufacturing resources including all kinds of service resources. The manufacturing resources formulate the standard interface component of cloud through unifying registration issued tools to match different demanders call; For NC machining equipment, instruments and meters, hardware service resources, could be used with a new type of intelligent terminals based on the clouds access, which realizes the convenient access clouds formation components offering user matching using different demand. (5) platform service component's function is to store and manage all kinds of thick granularity range service component, for different service demand. (6) service component layer as the middle transition of the business model and service component layer, form different thick size types of business by combining different service component for the different business model. (7) business model layer is the function of customer oriented demanders of the business needs of the business process definition, calls the different service component in response to the different needs of the business. (8) trade search matching layer provides the users' demand match seeking, and guide the transaction between cloud demand and cloud resources, records management, transaction process and 
give credit rating value. (9) the role of the user layer issues demand of all kinds of manufacture, participates in the service business, publishes industry knowledge and experience, etc.

In content networking environment and discrete manufacturing system PLM control platform of the system framework,for discrete manufacturing enterprise the whole life cycle of the product and import PLM system, introduce the content networking and visual management aspects of the technology and method, to construct a simple, intuitive for management decision-making as to show the enterprise operation process of the product as the center and the PLM in all kinds of real-time information; Provide control Suggestions, control effect prediction and simulation of visual control platform, as shown in Figure 1.

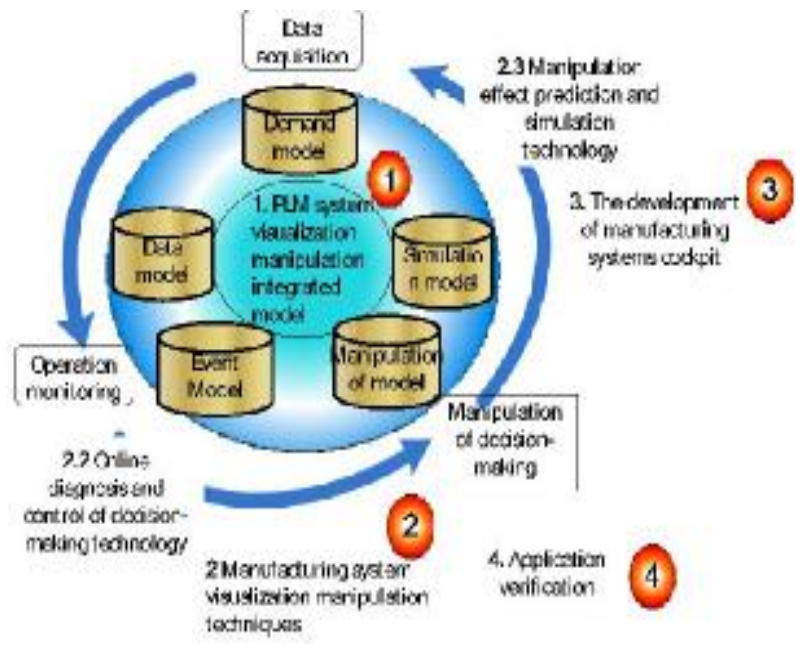

Figure 1 The principle diagram of visualization PLM control platform operation

From Figure 1, the visualization of PLM control platform operation principle has two most important parts of PLM system visualization and control integration model and PLM visual control technology, introduces in detail as following. Improving product quality, ensuring production schedule, optimizing the use of resources, reducing manufacturing cost, maintain the stability of system, improving the efficiency of the system, are the main goals of PLM system main goal ${ }^{[8,9]}$. According to these goals, research tie-in PLM system visualization control integration model, it includes demand model, data model, event model and control model and simulation model, etc. Including: demand model from the Angle of the control target and control content of PLM, describe the PLM visual control demand; Data model describe all kinds of data that meet the needs of PLM visual control, including data content, particle size, source, frequency, etc al.. In the Figure 1, the event model defines the PLM control events, and establishes the relationship between events and PLM data, which is the basis of analysis of PLM running status and diagnosis. The controlling model describes the events of the processing method, the control object and the control strategy. Simulation model is mainly used for predicting the effect of the control instructions and future state of the effects on the PLM system. Based on the analysis of PLM control goal and the content, the state of the PLM data and its ultimate source, external and internal events trigger PLM control and its processing method are analysed. The PLM visual control integration model is established which includes the need, data and events, manipulation, simulation, and other content and their interrelations shown in Figure 2. 


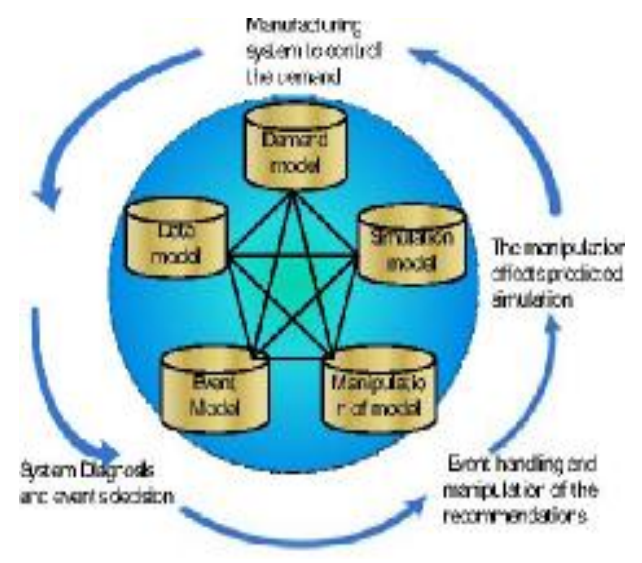

Figure 2 Integration of PLM platform visual control

Through Witness SIMBA SDK simulation optimization software, simulate the established PLM visualization simulation control integration model, check and improvement of the established model. For PLM data with multi-source, distribution and heterogeneity, mass and real-time characteristics, using the result of real-time database, distributed database, data fusion and data mining, visualization, virtual instrument/instrument technology research, according to the demand of PLM visual manipulation, to realize PLM data filtering, management and visualization technology.

\section{PLM platform engine control technology and management tools}

Discrete manufacturing system PLM control the construction of the platform is a huge and complicated project.In order to improve the development efficiency and quality of the platform, reduce the risk of platform development and development costs, and improve the level of standardization and platform of integrated platform. It need breaking through the above core platform and technology in line with the standards of the platform and system structure to develop a series of discrete manufacturing system PLM control platform common engine suite and management tool sets. The platform shows operators PLM the real-time status, control data needed for decision, diagnosis and control though a form way, and to simulate the plan given to ERP to improve the scientific nature of PLM control decision-making, achieve optimal operation of the PLM. On the basis of PLM control circuits "Information acquisition $\rightarrow$ operation monitoring $\rightarrow$ system control $\rightarrow$ information to obtain" , researching data management and visualization technology, PLM online diagnosis, control and decision-making and control effect prediction and simulation technology, to provide technical guidance for the construction of a PLM system, as shown in Figure 3.

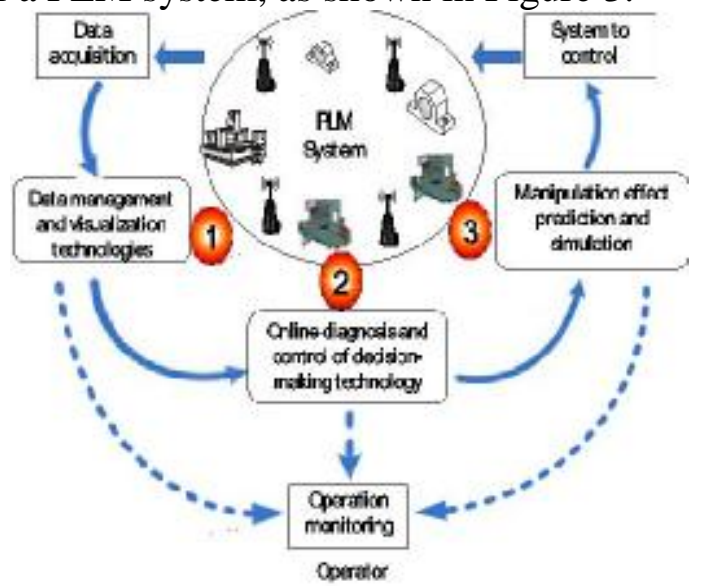

Figure 3 The technology roadmap of PLM visual control

(1) The PLM visual control technology and the key technology.

(a) data management and visualization technology, PLM system data is characteristic with multiple 
source, distribution and heterogeneity, vast sea, real-time characteristics.According to the control target of PLM and manipulating content, the platform can be used for filtering,organizing,storage, retrieval and visual displaying the data in the PLM.

(b) Online diagnosis and control decision technology: based on the data model, event model and control model, the PLM system status online diagnosis and control decision-making techniques, such as support for planning, decision-making, optimize configuration.

(c) Control effect prediction and simulation technology: based on the effect of simulation model and real-time data of the PLM system control, the future state of prediction and simulation technology, it helps to improve the PLM support the scientific nature of the control and optimization.

(2) The discrete manufacturing system PLM control platform of common engine suite. Discrete manufacturing system PLM platform to control the engine for the common platform development, application and popularization provides basic logic and service support, including main credit evaluation engine, trading synergy logical, intelligent matching engine and knowledge management, business logic polymerization and classification engine, etc al..

(a)Credit assessment engine of trade subject. The credit evaluation index based on manufacturing control platform, adopt feedback mechanism and main credit evaluation algorithm of manufacturing control platform, and realize the main trade credit information management, credit evaluation and comprehensive credit analysis of inquires.

(b)Intelligent matching engine. The manufacturing services search algorithm, through the way such as keywords, structure, and semantics to realize the quick search of manufacturing services, and then according to the function,, data, service quality and execution of manufacturing service, adopt the intelligent semantic matching algorithm to achieve intelligent matching of manufacturing services supply and demand.

(c)Business logic cooperation engine. Through manufacturing capability trade description language and management methods, make static description for the whole transaction process, realize the creation, operation, monitoring, exception handling, and evaluation of trading examples .

(d)Business logic management engine. Based on the multilevel and multidimensional comprehensive modeling of the discrete manufacturing system manufacturing capability of the trading process, combined with the process, the similarity measure algorithm and process model retrieval algorithm, and the corresponding simulation analysis technology analysis, the structure rationality, transaction process behavior compliance, activities can be scheduling, process success rate were analyzed, which realize the online trading process evolution and management.

(e)Knowledge polymerization and classification engine. Based on data mining and analysis, industry knowledge polymerization and classification algorithm, using web crawler, NLP (nonlinear programming), to realize the establishment of manufacturing knowledge base, semantic analysis, semantic search and query.

(3)The discrete manufacturing system PLM control platform common management tool sets. The common management tool set of discrete manufacturing system PLM control platform are mainly to provide user-friendly man-machine interactive application tools for users, realizing the function of the operability of the platform and convenience, including unified user management, business management system, system management, and resources virtualization tools etc al..

(a)Unified user management. Support cross classification, industry classification, support for single sign, meet the needs of security,unity and extensibility.

(b)Business management system tools. For the coordination of the main body of the customizable outsourcing business to provide total life cycle management function, including the definition, management, intelligent recommendation, analysis, simulation and improvement of outsourcing template, support for the deployment, implementation, monitoring, diagnosis, evolution and optimization of outsourcing case.

(c)System management tools. Support to build more tenants, large capacity, high concurrency, high availability of the control platform to provide various management functions. 
(d)Resources virtualization tools. Realize the mainstream computing resources, storage resources and network resources, manufacturing resources, and other physical hardware and software resources of virtualization centralized management and monitoring.

\section{Discrete manufacturing system PLM control platform of service mode}

How to aim at the study of discrete manufacturing system manufacturing services business characteristics and manufacturing services resources characteristics research and establish feasible operation service mode is an important problem for the need to break through discrete manufacturing system PLM control platform to enter the stage of practical application, realize the integration and sharing of manufacturing resources. Based on discrete manufacturing system characteristics and requirements, combined with the providing way of discrete manufacturing system manufacturing services provide way, the types of manufacturing service resources, the structure mode of manufacturing participate in role structure mode, manufacturing services business and profit model, around the "scattered resources concentration, focus resources scattered service" manufacturing ideas, as shown in Figure 4.

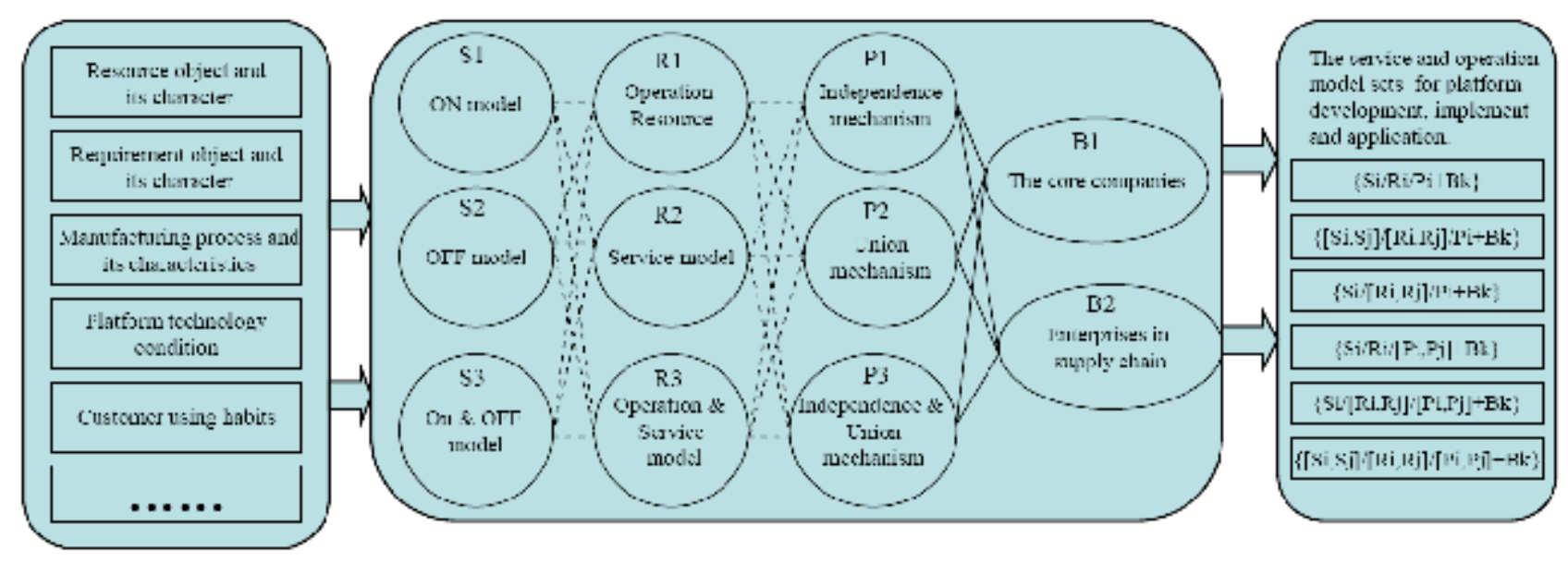

Figure 4 the service ideograph of PLM system platform of discrete manufacturing system

In Figure 4, "222" include: pure manufacturing services online (ON mode), combination of online and offline manufacturing service (ON\&OFF mode) two kinds of typical manufacturing services provide way; $R$ service resources and $S$ service resources two typical types of manufacturing services resources; Three parties (refers to resources providers, resource demanders, disclosing party platform operator tripartite) manufacturing services and four (refers to the resource provider, demanders of resources, resource integration service, platform operator sifang) participate in role of manufacturing service two kinds of typical structure mode. " $+2 "$ means more than a typical manufacturing service model based on the core technology support tools can be taken charge mode and membership fee model two kinds of typical business and profit model.

The core company provides technology of platform sets and manufacturing resources to make further personalized combination and configuration, in order to guide the development, implementation and commercial operation of the corresponding PLM discrete manufacturing system control platform.

\section{Literature References}

Concerned the management of Internet of Things, the visualization of product lifecycle management, controlling modeling, and controlling simulation, are having been attracted a lot of researchers. For example, Professor Qi Guoning of Zhejiang university studied the project management of product life cycle management[1]. Wang Chengen of Northeastern university studied the modeling and management of product life cycle[2] . Josef Scho ttner of Munich industrial university studied 
manufacturing enterprise's product data management principles, concepts, strategies[3], and so on. In the present research, the research object based on the mathematical model control method and additional assumptions are often far away from the actual situation and it is difficult to be used in complex PLM effectively. For the limitations of conventional intelligent method and simulation technology, some technology companies have invested considerable human and financial resources, combined with the particular goals in the PLM, promote research and development of automation technology, have achieved some practical results, but these studies are lack of universality[4-6]. Particularly it is hard for production conditions changed frequently in manufacturing enterprises. So it is urgent that actual demand of complex PLM control and optimization is studied combined with the intelligent method, optimization theory. It is needed to study optimization arithmetic of high performance, confirm and apply it in the typical complex PLM, lay a solid foundation for the development of PLM modeling and optimization software which has our country's independent property rights in the networking environment, in order to improve the overall level of the automation of manufacturing industry in China.

\section{Conclusion}

Discrete manufacturing system PLM control platform will become an important supporting means of discrete manufacturing system in our country to make full use of and sharing of manufacturing resources, and enhance the comprehensive competitiveness of enterprises, which also is an important development direction needed to explore in the field of advanced manufacturing in China. The research content of discrete manufacturing system PLM control platform key common technology platform, includes the core theory and technology of platform, the standards and specifications of platform, the common engine and common management tool, the structure platform system, the service platform and operation mode. Discrete manufacturing system PLM control platform has the connotation and characteristics including participate in resources advantage, the multidimensional nature of resources providing manufacturing services, service trade.

\section{Acknowledgement}

This research is sponsored by Foundation of Anhui natural science research in colleges and universities (KJ2015A303) projects), China Postdoctoral Science Foundation(No. 2013M541826) and Advance research program of state funds of AHPU (zryy 1312).

\section{References}

[1] SU Shao-hui, QI Guo-ning, GU Qiao-xiang, JI Yang-jian, CU I Jian. Project management integrated model for product lifecycle management[J]. Journal of Zhejiang University (Engineering Science), 2008,42(11):1951-1956

[2] SHU Qi-lin,WANG Cheng-en.Manufacturing Information Model During the Product Lifecycle[J]. JoumalofNortheasternUniversity, 2005, 26（8）: 774-777

[3] WANG Shu-ying.Research on authority controlmodel for industrial-chain coordinate business platform[J]. Application Research of Computers, 2010,27 (1) :170-173

[4] GU Yuqi, WANG Zhengxiao, PAN Xiaohong. Research on the asp platform for networked manufacturing in textile region[C]//The 2nd International Technology and Innovation Conference. London, UK: IET, 2009:1118-1122.

[5] SUN Xiao,ZHANG Xiu-zhi,WANG Xiu-ying. Networked sharing platform of enterprise-group-oriented manufacture resources[J]. Journal of Jilin University, 2009,39(1):222-226. 
[6] YIN Sheng, YIN Chao, LIUFei, GUOKun-yi. Integrated service mechanism of networked collaborative product development resources[J]. Computer Integrated Manufacturing Systems, 2009,15(11):2233-2240.

[7] ZHANG Li, YIN Guo-fu, WANG Wei, DENG Zhi-tao. Research on ASP-Oriented Collaborative Analysis Service Technology in Network Environment[J]. JOURNAL OF NORTH UNIVERSITY OF CHINA, 2008,29(2):136-141.

[8] Dillon T, Chen Wu, Chang E. Cloud computing: issues and challenges[C]//Proceedings of the 24th IEEE International Conference on Advanced Information Networking and Applications. Washington, DC., USA: IEEE, 2010:27-32.

[9] Atzori Luigi, Iera Antonio, Morabito Giacomo. The internet of things: a survey [J]. Computer Networks, 2010, 54(15):2787-2805

[10] Yan Wang;Zhicheng Ji.Energy efficiency quantitative analysis method of discrete manufacturing system[J].Modern Physics Letters B.2017,Vol.31(No.19-21): 1740071

[11] Y. Qamsane;M. El Hamlaoui;A. Tajer;A. Philippot.A Tool Support to Distributed Control Synthesis and Grafcet Implementation for Discrete Event Manufacturing Systems[J].IFAC-PapersOnLine.2017,Vol.50(No.1): 5806-5811.

[12] Ana Paula Estrada-Vargas;Jean-Jacques Lesage;Ernesto López-Mellado.A stepwise method for identification of controlled discrete manufacturing systems[J].International Journal of Computer Integrated Manufacturing.2015,Vol.28(No.2): 187-199. 